\title{
'He was obliged to seek refuge': an illustrative example of a cross-language interview analysis
}

Erika Kalocsányiová, Institute for Lifecourse Development, University of Greenwich Malika Shatnawi, Polytechnic Institute Australia

This paper provides one of the first inquiries into the interactional dynamics of an interpreter-mediated research encounter. All spoken interactions - i.e. originals and real-time translations produced in a multilingual interview conducted with a Syrian refugee - were audio-recorded, transcribed verbatim using CA notation, retranslated and collaboratively analysed from three major perspectives: common language, equivalence, and loss and gain in translation. A stimulated recall interview, field notes and audio-recorded work sessions documenting our interpretative practices complement the data. Fixing our analytical gaze on the minute details of language use across English-Arabic allowed for a novel inquiry into specific moments of meaning making, role performances and rapport-building in qualitative interviewing. Our examples illustrate how an agreed-on sense of the source meaning is established not only during the interview itself, but also at the point of its multilingual representation and analysis.

Keywords: interpreter-mediated interview, multilingual research, loss and gain in translation, collaborative analysis

\section{Introduction}

The forces of globalization have exacerbated the need for developing a reflexive multilingual research practice. Researcher positionality, work in multilingual research teams, building of relationships in the field and the ethical implications of language choices, as well as the language politics of representation and dissemination have received considerable attention since the 2010s. Previous studies have also provided some critical reflections on including interpreters in qualitative cross-language research (Andrews, 2013; Berman and Tyyskä, 2011; Resch and Enzenhofer, 2018), and a compelling body of literature has emerged around the theme of public service interpreting in health sciences, police interviews and refugee status determination procedures (e.g. Gibb and Good, 2014; Määttä, 2015; Brämberg and Dahlberg, 2013). So far, however, most scholars have focused on power asymmetries and codes of conduct, whilst methodologically rigorous explorations of interpreter-assisted crosscultural encounters are still lacking.

This paper provides one of the first inquiries into the interactional dynamics of an interpreter-mediated research encounter. In the pages that follow, extracts from a multilingual 
interview (Arabic, English and French) are presented and discussed from three major angles, each of which is associated with much-disputed subjects within interpreting studies: common language, equivalence, and loss and gain in translation. The interview was conducted as part of ethnographic research designed to understand the impact of multilingualism on forced migrants' integration trajectories. It lasted approximately an hour, and it involved the researcher (first author), the interviewee (a Syrian man who sought asylum in Luxembourg) and a research assistant who assumed the role of English-Arabic interpreter. All spoken interactions produced in this encounter - originals and translations - were audio-recorded and later transcribed verbatim using conversation analysis ${ }^{\mathrm{i}}(\mathrm{CA})$ notation. A detailed and intricate analysis of turns, topics and patterns was then performed to make transparent the contributions of the researcher, the interviewee and the interpreter in the production of meaning. Audio-recorded discussions with the interpreter and co-researcher (second author) about our shifting understandings of words and concepts complement the data.

The paper outlines a proposal for how to approach the analysis of cross-language interview material. Our approach is a descriptive one: rather than giving oversimplified directions or tools for analysis, we aim to provide food for thought and contribute to an emerging understanding of language barriers and translation dilemmas in research. We begin with a brief overview of the roles that are generally ascribed to interpreters/translators in research. Next, our research process and methods are discussed. Close attention is given to the background and individual contribution of each person involved. The remaining parts of the paper are organized around the three key themes established above: common language, equivalence, and loss and gain in translation. Each area is discussed in detail using illustrative evidence from the transcription.

\section{Interpreters in research: from conduits to active producers of knowledge}

In an effort to advance knowledge about super-diverse societies, a growing body of research seeks to document a diverse range of voices and experiences. Studies that depend on third parties to communicate across languages have become the rule rather than the exception. Yet, the predominant approach in cross-language research is still to conceal the interpreter and/or treat interpreting as a mechanical and potentially problematic part of the research process: '[within the positivist paradigm] researchers who conduct cross-language research continue to seek ways to control for the 'effects' of the interpreter/translator, to treat them as a threat to validity, and to make them invisible in the process and product' (Berman and Tyyskä, 2011: 179). As a consequence, interpreters are imagined as 'neutral mouthpieces' (Temple and 
Edwards, 2002: 10), transmitters or 'conduits of communication' (Freed, 1988), and 'faceless voices' (Morris, 2010: 20) who should not embellish, add or subtract from what is being communicated. Researchers working from this perspective generally discuss validity in terms of correctness and completeness. Elimination of bias occupies a central position within this paradigm, as illustrated by Keselman et al. (2010), a study using interpreter-mediated interviews with asylum-seeking minors. However, the above notions have long troubled researchers who work with a critical understanding of language as non-transparent, of meaning as situated, and of the power of interpretation as fundamental to meaning making (Ross, 2010).

The research presented here is situated within this second paradigm, in which the interpreter is acknowledged as active producer of research data. As von Glasersfeld (1983: 207) has argued, 'the activity of interpreting [be it within or across languages] involves experience, the coordination of conceptual structures, and symbolic representation; that is to say, it involves the very activities of cognition and thus, inevitably, a theory of knowledge'. Like researchers, interpreters bring their assumptions, accumulated experiences, and concerns to the research process: they infer the thoughts and intentions of speakers by reference to their own understanding of the concepts discussed. In fact, interpreting an utterance (or a written piece of language) requires 'the insertion of whatever we consider its conventional meaning into a specific experiential context' (von Glasersfeld, 1983: 212). Qualitative interpreterassisted interviewing thus becomes subject to multiple subjectivities (Temple and Edwards, 2002; Temple and Young, 2004). Similarly, translators leave their mark on research when transforming interview transcripts from one language to other. As translation is also an interpretive act, meaning may be lost or (re-)created in the process (Fersch, 2013). Finally, Oliver et al. (2005) direct our attention to the complexities of transcription itself, arguing that transcribers hear interviews through their own cultural-linguistic filters. To pursue these nuances of knowledge production, we move on to describe in greater detail the position of each contributor within the research process.

\section{Background to the research context}

The interview discussed here has been extracted from a larger ethnographic dataset, which was collected for the purposes of the first author's doctoral research. This project set out to investigate the complexities that define forced migrants' linguistic integration efforts in multilingual societies. For a period of two years, the first author followed the trajectories of five men from Syria and Iraq who had been granted international protection in Luxembourg 
(see Kalocsányiová 2017, 2020 for examples of this work). The interview was the first in a series, and it was designed to elicit information about the research participant's linguistic repertoire, migration experience and language (hi)story. A number of broad, open-ended questions were asked in order to ensure rich qualitative data. The encounter started in French with the exchange of pleasantries between researcher and participant. Afterwards, the interpreter was brought in to translate the elicitation questions from English to Arabic. The interviewee replied in Arabic, which was translated back into English in the form of third person summaries. Note that the interpreter was encouraged to use the third person rather than first-person interpreting. This was to mark the utterances as in part constructed by the interpreter.

\section{Participants of the interview}

The participant/interviewee was a Syrian man in his early forties. In 2015, he fled Damascus and took refuge in Luxembourg. To ensure confidentiality, we will refer to him by the pseudonym 'Ram'. Ram is Arabic speaking, but at the time of the interview he had accumulated a fair knowledge of French since arriving in Luxembourg. He also had some receptive competence in English, but he expressed a preference for conversing in Arabic. His experience of forced displacement had made him familiar with the routines and procedures of interpreter-mediated discussions. At the time of the interview, Ram knew the researcher for about ten months through her fieldwork, which was undertaken as part of a larger 4-year doctorate. The researcher, whose first language is Hungarian, has an MA in translation and interpreting studies. Of the languages relevant in the interview, she had knowledge of English and French. She also had prior experience in 'juggling' multiple languages in professional and research contexts. A former translator and communication graduate from the researcher's university volunteered to act as interpreter between researcher and interviewee. The interpreter was born in Morocco, but he lived in the United Kingdom, Luxembourg and France for a considerable time. He had formal education in qualitative research methods and ethics. The research focus and the discipline's jargon were also known to him. He was not a stranger to Luxembourg's complex sociolinguistic environment either. To help with the interpretation of data, the first author also invited a second researcher to collaborate on the project: the second author, who was born and educated in Jordan, is a translation and TESOL specialist. All participants thus had extensive experience of 'living across languages and being involved in the dilemmas of translated worlds' (Temple and Young, 2004: 162), and each brought a unique perspective to the process of analysis. 


\section{Procedure}

The interview was audio-recorded in February 2017. It took place in a café and lasted approximately 59 minutes. Shortly afterwards, the researcher wrote up her field notes about the context, nonverbal behaviours of the participants, and personal impressions of the encounter (ease of discussion, feelings and moments that stood out as significant). In line with the recommendations offered by Williamson et al. (2011) and Andrews (2013), the interpreter was also interviewed about particular aspects of the interview process: the questions were aimed to check impressions and seek cultural insights. Subsequently, the researcher transcribed the elicitation questions and the interpreter's verbal summaries of the participant's responses and comments. These were screened for patterns and themes relevant to the research questions of the larger project. To make the production of meaning more transparent, the interpreter-participant interactions in Arabic were also cross-checked, transcribed and translated verbatim. Thus, we produced a written version of all interaction in all languages. The co-author, who did the second round of transcription, also pinpointed areas and patterns to consider in more detail. The new comprehensive transcript was subjected to an intricate analysis: the authors jointly constructed a shared interpretation of the interview material. The interactional mechanisms were analysed bearing in mind the overall interview data and the contextual clues from the researcher's field notes.

All work sessions were audio-recorded to capture our interpretative practices and reflections about the data. These recordings document the many ways in which our different trajectories, beliefs about language and forms of cultural knowledge influenced the research process. In addition, the interpreter was asked to undertake a stimulated recall interview (Dempsey, 2010). He was given access to the original audio file and was shown the full transcript. He was then invited to share his observations and to answer specific questions arising from our analysis. His responses both corroborated and challenged our findings. In the transcription process, CA conventions that include timed pauses, overlaps, emphasis, paralinguistic features and annotations were utilized. The key to the transcription conventions is given in appendix 1. In the extracts below, initials are used to indicate the participants: interviewee (R), interpreter (Y) and researcher (E). English translations are provided in italics. Having described our framework and methods, we now move on to the discussion of our findings. These will be treated under three headings conforming to the three major angles of analysis: common language, equivalence, and loss and gain in translation. Clearly, there is a strong interdependence and overlap between these themes; however, we discuss them 
separately to expose some of the less obvious, yet crucial aspects of meaning construction in interpreter-mediated research encounters.

\section{Speaking a common language}

Language is one of the fundamental tools for conducting ethnographic research. After all, much of the fieldwork, conversations and interviews through which the data are collected depend on a shared language. However, this standard of common language no longer holds in globalized or super-diverse contexts, which require the researcher to work in multiple languages and often with the assistance of an interpreter/translator. In fields of intercultural studies, scholars have recommended matching interpreters and interview participants based on their main/preferred language and cultural 'sameness' (e.g. Merriam et al., 2001). It is a process that sounds straightforward but is fairly complicated in practice. Firstly, dialectal variation constitutes a major challenge for interpreters who translate for forcibly displaced persons. Let us take Arabic as a case in point. Arabic is the official language of over 20 countries from Northwest Africa to the Persian Gulf. In spoken form, it is a dialect-rich language and speakers from different regions cannot always easily understand each other (TWB, 2017). Our ethnographic data support this claim: the research participants, who are speakers of Levantine colloquial, struggled on more than one occasion to communicate with the public interpreters available in Luxembourg. Since the beginning of the refugee 'crisis' in 2011, numerous interpreters were recruited from among heritage speakers, who, however, tend to experience more difficulties in lexical selection and grammatical accuracy (Albirini, 2014). Secondly, even speakers of the same dialect often have notably different vocabularies and language habits at the level of register, style, pronunciation and so forth (Bellos, 2011). Thirdly, as Shklarov (2007: 531) points out, '[p]eople who routinely perform translations are, most often, long-time immigrants and have been educated in the Western tradition'. This makes them, to some extent, culturally distant from other members of their speech community. Fourthly, Williamson et al. (2011: 383) caution that the comfort that can result from matching interpreters and participants on certain characteristics (potentially) limits the breadth and depth of interview data: '[t]aken-for-granted assumptions [...] can act as blinders, preventing interpreters from probing certain topics and/or limiting the depth and detail that participants offer in response to interview questions'. Difference can be a barrier as much as a stimulator of communication. Finally, participants and translators/interpreters in forced migration research and other cross-national studies are almost always mobile subjects who engage with a broad variety of groups, networks and communities. Most of them inhabit 
multilingual spaces, where culture is no longer a fixed and monolithic phenomenon shared (solely) by a given community (Kramsch, 2014).

As an illustration of the above considerations, let us consider the following example. At the start of the interview, the interviewee $(\mathrm{R})$ and the interpreter $(\mathrm{Y})$ engaged in a variant of a 'where are you from' discussion:

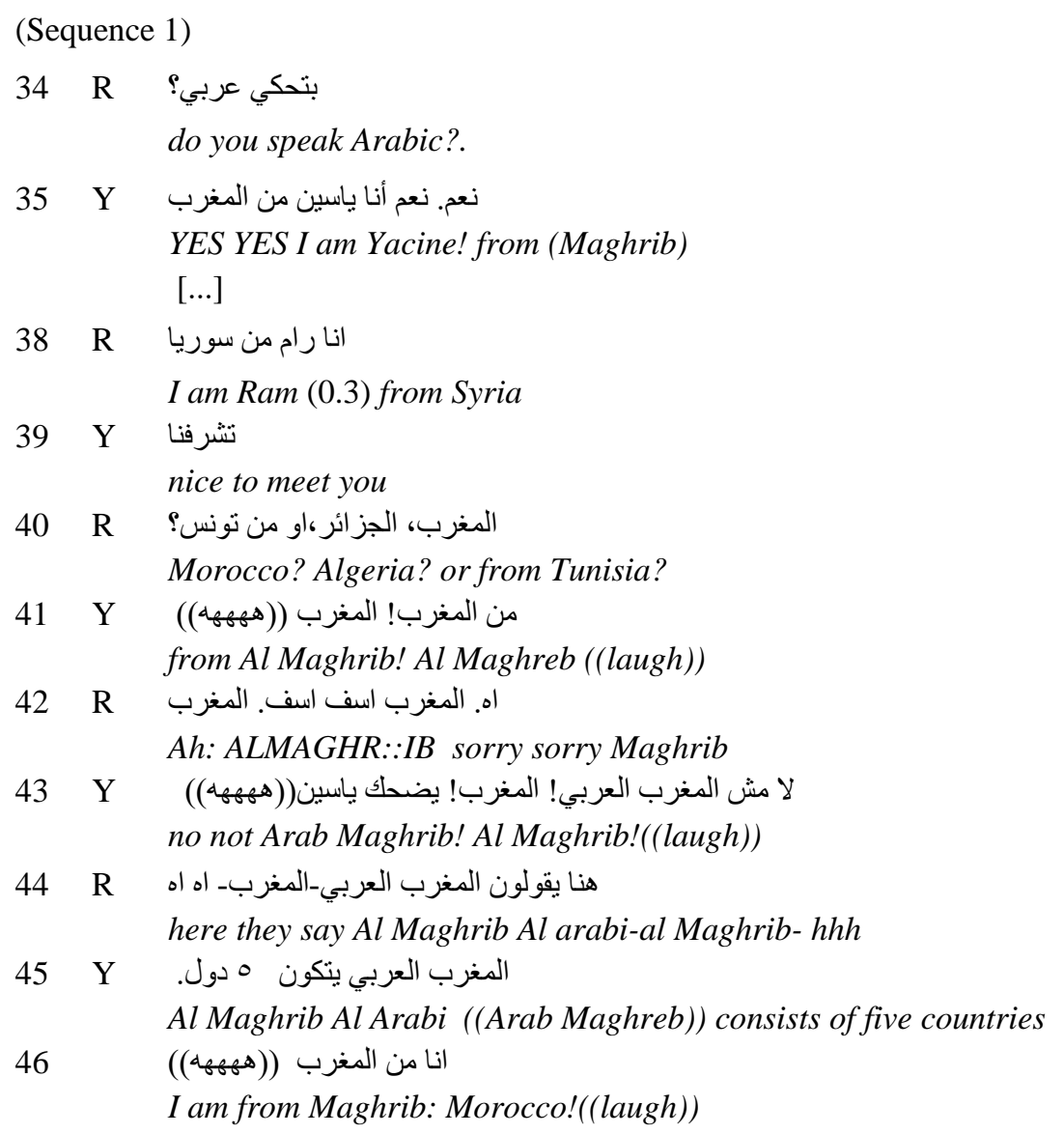

On the surface, this brief exchange of information about ethno-national origins could be considered an expression of genuine curiosity and an effort to connect (i.e. a conversation starter) or, alternatively, a factual inquiry into the interpreter's place of origin. However, as a more sophisticated analysis reveals, the above conversation also allowed for a negotiation of dialectal differences, which became instantly evident with the introduction of the toponym 'Maghreb' (line 35). The Arabic term 'maghrib' means the West or the Land of the Setting Sun. Geopolitically, the area of Maghreb (also spelled Maghrib) is taken to include the Atlas Mountains and the coastal plain of Morocco, Algeria, Tunisia and Libya. This first meaning coincides with Ram's interpretation of the concept, as suggested by his question in line 40 ( Morocco? Algeria? Or from Tunisia?). In some Arabic dialects, Maghreb is also an abbreviated form of al-Maghrib al-aqsa - the far West - meaning Morocco only (Kogelmann 2010). This second meaning overlaps with the interpreter's 
understanding of the term, as demonstrated by the emphatic repetition of the expression in lines 41, 43 and 46. In contrast, in English the use of the term Maghreb is limited to its first meaning (i.e. Northwest Africa), raising the question of how to translate and transcribe the aforementioned sequence without suppressing the polysemy which - as we have seen - was the source of a misunderstanding. After considering several translation solutions, we opted for the denomination Arab Maghreb in English (translit. al-Maghrib al'Arabi) to preserve the polysemy, while at the same time keeping the difference between the two meanings marked.

The interpreter-participant match in terms of sociodemographic characteristics is also regarded an important factor in the literature: within the different hierarchies of suitability, particular stress is placed on age, gender, religion and class (Freed, 1988; Tribe and Keefe, 2009). Commonly noted benefits of matching are that research participants perceive interpreters as trustworthy, which, as stated by Williamson et al. (2011: 383), 'can facilitate the development of rapport and enhance participant's comfort with the interview experience'. Recognising and relating to one's means of expression can be a fast track to rapport building. An example of how this is achieved is shown below:

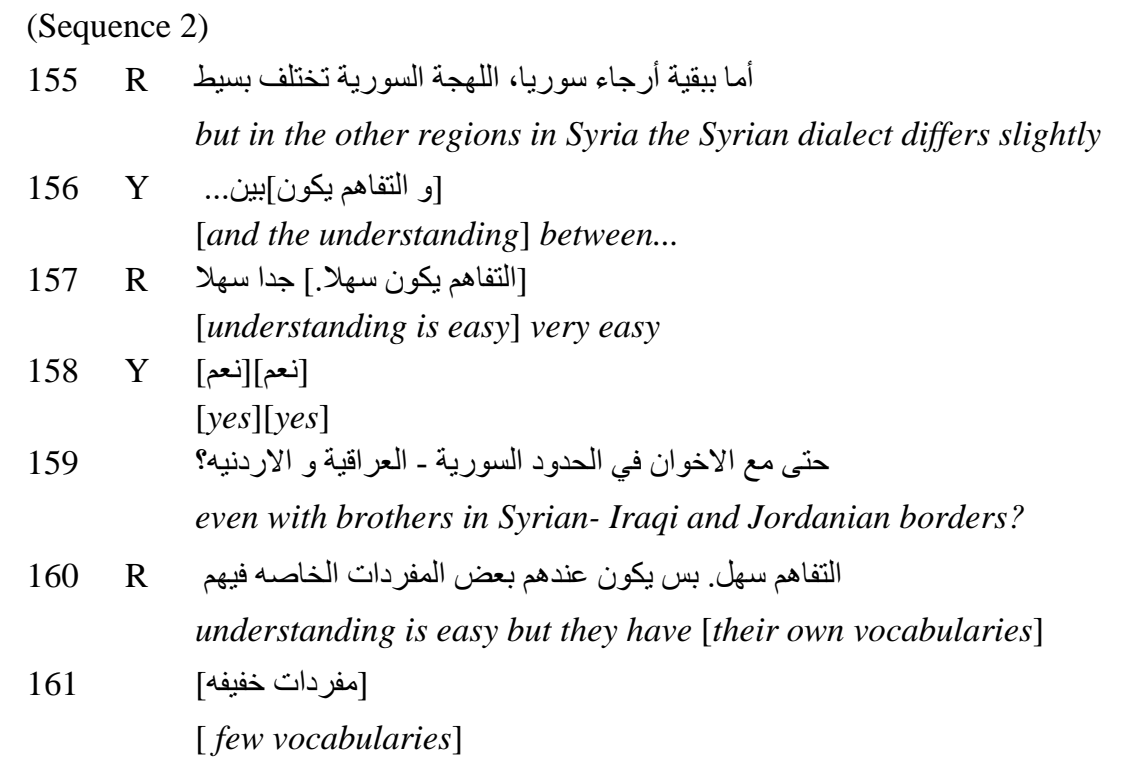

In this sequence, the expression brothers (الإخوة) deserves attention. The use of kinship terms in order to refer to non-kin is a widespread phenomenon in the Arabic-speaking world. Referential as well as vocative uses of $a k h i$ // أخو (lit. brother) generally carry positive connotations: it is a respectful (or even endearing) way to address and/or speak about nonrelated individuals. In terms of perception, the interpreter's word choice - which had no counterpart in the original - is arguably an active attempt to move from being an outsider to an 'inbetweener' (Milligan, 2016). As the recall interview revealed, this transition towards the 
use of more culture-specific expressions was, indeed, an interpreting strategy to put the participant at ease. The above observations are consistent with previous studies; however, we would caution any reader against jumping to the conclusion that communication and, especially, interpreting are ipso facto better on the basis of sociodemographic correspondence (cf. Temple and Edwards, 2002). An interactionally grounded analysis - as shown above - is needed in order to investigate rapport as 'emergent and observable conduct' [emphasis in the original] (Prior 2017: 4) in cross-language interviewing.

Let us now turn to the researcher-interpreter interactions, which fall into what can be termed as intercultural communication in English as a lingua franca (ELF) ${ }^{\mathrm{ii}}$. As speakers of ELF, the researcher and interpreter had to work out - as House (2014: 365) put it - 'a new joint linguistic, intercultural and behavioural basis [for communication]'. This also influenced the interactional dynamics: for instance, at times, the interpreter would check in with the researcher to seek clarification or to consider alternative formulations. Our analysis has also shown that the interpreter often reformulated Ram's responses using a higher register ${ }^{\text {iii }}$, more consistent syntax and specialist terminology in English. Drawing a parallel with the theoretical work of Bellos (2011), we argue that the interpreter raised the social register of the source a 'notch or two':

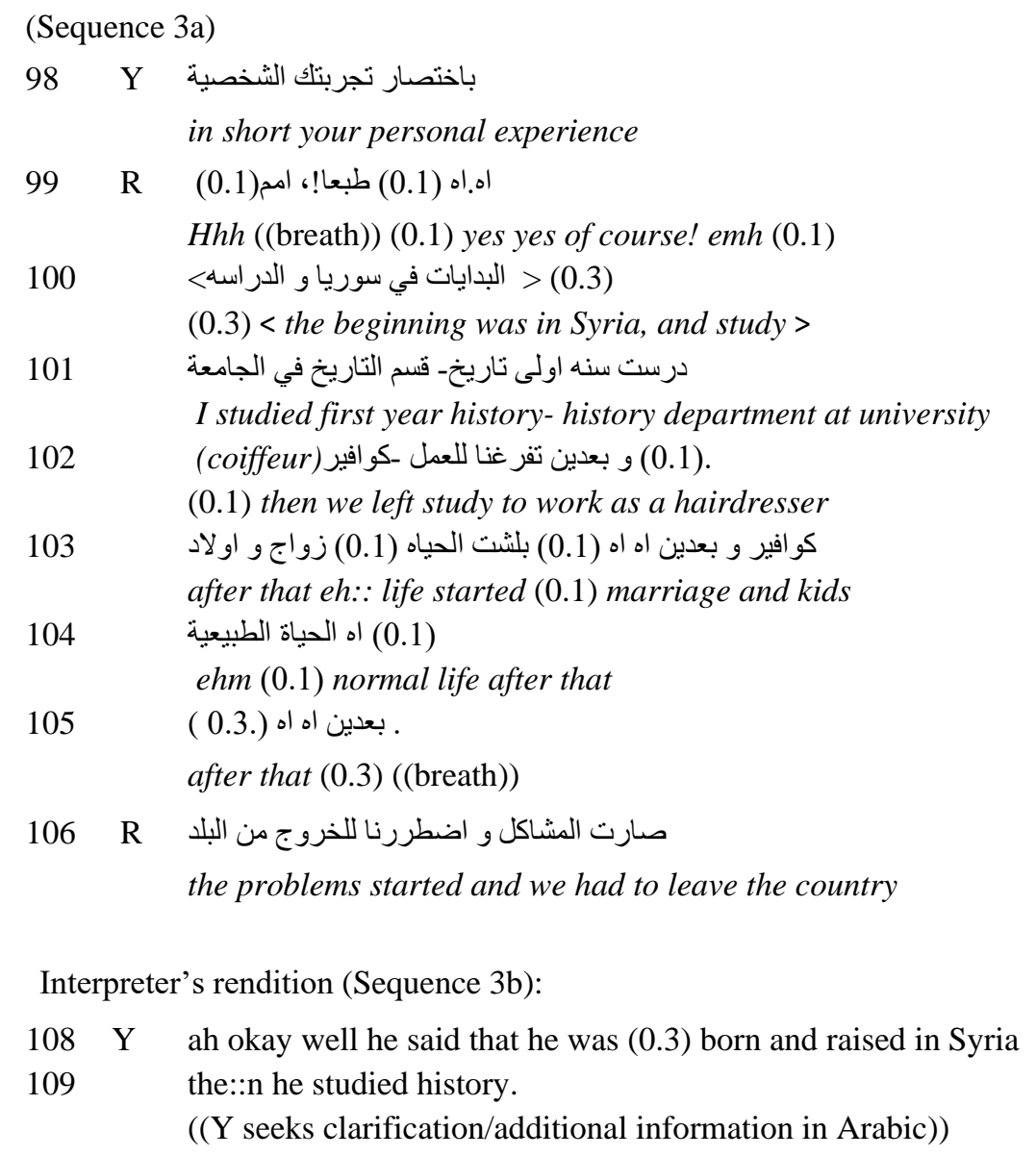


113 ehm:: he started university ehm at the department of history

114 he did one year in history (0.2) at the university then

115 he left to work and he worked as a hair[dresser]

$116 \mathrm{E}$

$117 \mathrm{Y}$ > yeah yeah< for (1.0) three years yeah then after what happened in

118 Syria he was obliged to (0.5) seek refuge in another country

From the comparison of these two sequences ( $3 a$ and $3 b$ ), it is immediately apparent that the interpreter's rendition adheres more strongly to a normalized idea of language. Bellos (2011: 151) associates similar shifts with '[the] seemingly inevitable bias against representing forms of language recognized in the source culture as regional, uncouth, ill-educated, or taboo by socially matching forms in the target tongue - presumably because doing so risks identifying the translator as a member of just such a marginal or subordinated class.' However, in our view, such creative shifts between registers also work as an interactional resource through which the interpreter ensures inter-turn cohesion and topical continuity. In order to support mutual understanding, the interpreter makes continuous adjustments in vocabulary, syntax and tone to align himself with the speakers as the dialogue unfolds. Evidence of this can be found in lines 108 and 118 of the interpreter's rendition, where Ram's turn is made more explicit through a fuller description and paraphrasing (the beginning was in Syria $=>$ he was born and raised in Syria; had to leave the country $=>$ obliged to seek refuge in another country). In dialogic environments, there is often an expectation towards interpreters to make the conversation (appear) planned and goal-oriented with regard to the thematic structure (Wadensjö, 1998). Yet, academic researchers are not used to this 'dependency' and, consequently, concerns about trustworthiness and data quality often arise (Edwards, 2013). How these relate to the question of equivalence as well as loss and gain in translation is addressed in the next two sections.

\section{Chasing equivalence}

Up until the second half of the twentieth century, theories of written and spoken translation seemed locked in a debate over the triad of literal, free and faithful translation ${ }^{\text {iv }}$ (Munday, 2001). Nida's (1964) introduction of the concepts of formal and dynamic equivalence ${ }^{\mathrm{v}}$ in the effect was crucial in introducing a recipient-based orientation to translation theory. Despite being a controversial concept, equivalence has been of particular concern to scholars as it is inextricably linked with both definitional and practical aspects of translating/interpreting. One of the useful lessons to be drawn from a reflexive examination of the vast literature on equivalence is that there is no one correct rendition of speakers' turns. Bassnett's (2013: 79) 
analogy comparing translators to 'Aladdin in the enchanted vaults' applies as much or even more so to interpreters who formulate their translations in a flash. Following this line of thought, let us compare Ram's view on his employment perspectives and its subsequent rendition by the interpreter:

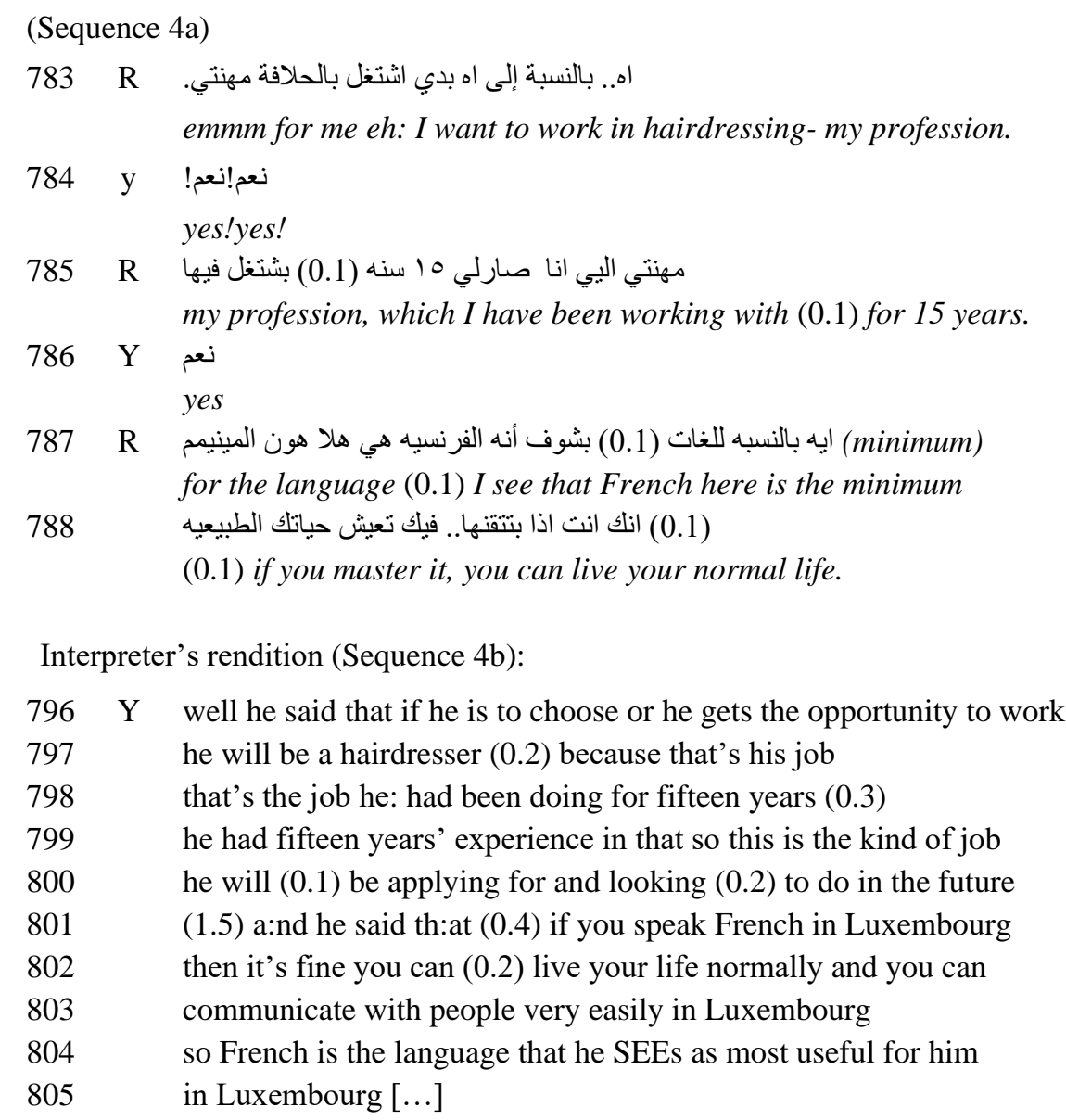

Ram's utterances (sequence 4a) are fairly straightforward, yet the interpreter's transformation into English still hints at the "dazzling array of possible word combinations that could be used to convey meaning' (Temple and Edwards, 2002: 3). What also calls for attention are the interpreter's somewhat redundant formulations: throughout the sequence, he repeatedly introduces various forms of lexical and semantic repetition ${ }^{\mathrm{vi}}$ (e.g. in lines 798-799: that's the job he: had been doing for fifteen years $\approx$ he had fifteen years' experience in that), backtracking (in line 796: if he is to choose was paraphrased into he gets the opportunity to work producing a repetition with variation) as well as explicating additions (lines 803-805). There is a clear tendency on the interpreter's part to explicate more than what a sense-forsense translation of the source text would call for. Under normal circumstances, interpreting is, in fact, more prone to text condensation, i.e. lexical and syntactic compression and strategic information reduction (Pöchhacker, 2004: 134). As a rule, interpreters are 
encouraged to avoid not only hesitations, filled pauses and cut-offs but also repetitions that are deemed 'unnecessary' (Straniero Sergio, 2012). Thus, in the field of interpreting studies, most of the speech elements reported above would be seen as disfluencies in output and/or coping tactics to compensate for cognitive overload and reformulation difficulties in the target language. In terms of style and quality, however, these insertions also serve as an interactional resource for clarifying ambiguities and making complex passages more accessible and intelligible for the speakers. It is worth noting that repetition, parallelism and paraphrase are all crucial structuring and persuasive discourse devices in Arabic rhetoric (Jawad, 2009). It is not unreasonable to assume that this played a role in the production of the interpreter's turns. When queried about substituting the speakers' turns with fuller descriptions, the interpreter attributed this translation behaviour to a preoccupation with ensuring that the research aims are met. Interestingly, in his corpus-driven research of interpreter-mediated talk shows, Straniero Sergio (2012: 43) reached a similar conclusion: '[t]he interpreter's tendency to say more than what the guest has said and/or to be more explicit is due to the constant concern to ensure that answers meet the host's expectations and/or confirm what s/he said in the question'.

In our tripartite research interview, the interpreter like other real-time users of language made inferences about the communicative intensions of the researcher/participant and transmitted $a$ version of what had been said. Building on Mason's (2006) research on dialogue interpreting, we argue that the interpreter's output traces how the different questionanswer turns were understood, i.e. the process of actual inferencing which is dependent upon what Widdowson (2004) calls pretext or perlocutionary purpose. As he aptly puts it, '[w]hat is relevant in text is what the users choose to make relevant in relation to what they are processing the language for' (p. 76). From the interpreter's rendition of the last sequence, it is safe to assume that he deemed the clue about speak[ing] French in Luxembourg (lines 801$805)$ relevant. This is evidenced by the introduction of elaborative utterances which had no counterpart in the original, such as you can communicate with people very easily in Luxembourg or French is the language that he SEEs as most useful for him. The insertion of the former is most likely attributable to the interpreter's (ambition to show his) profound understanding of the research context, while the latter is aimed at furnishing an explicit answer to the researcher's question. With these additions, the interpreter re-appropriated his role as active producer of the research data. On multiple occasions, he also re-used lexical items contained in the elicitation questions (and responses), thereby creating greater inter-turn coherence. Note that besides being a major coherence device, context recycling also eases the 
cognitive load of making a choice from among the available target language equivalents (cf. Straniero Sergio, 2012). In her study of pragmatic meaning in court interpreting, Jacobsen (2004: 247) categorises the aforementioned forms of text expansion as 'additions with significant impact on the semantic and/or pragmatic content of the source texts' and attributes them to '[interpreters'] instinctive judgment that end receivers would not be able to spot the presence of implicit information' and 'their preoccupation with building and conveying a mental model of speaker meaning'. Whereas additions (with impact) violate the standard rules and canons of judiciary interpreting, in qualitative cross-language interviewing these constitute a methodological rich point which makes salient the interpreter/translator's assumptions and advances our understanding of meaning-production. The interpreter is the first to tap into the participant's thoughts and experiences, and his reconstruction of the speaker's voice can both enrich and obscure the intended meaning.

\section{Loss and gain in translation}

The extent to which interpreters should deliberately contribute to the production of research accounts (through, for instance, asking interview questions and following up with probes) has been a controversial and much-disputed subject within the field. As noted by Murray and Wynne (2001), interpreters' attempts to direct the interviewing process does not compromise the authenticity or validity of the research account per se - on the contrary, it can enable the clarification of understanding. However, they also caution that ' $\mathrm{n}] \mathrm{ot}$ all (if many) interpreters will have the requisite knowledge and skills to combine interpreting, the crafting of pertinent research questions and the pursuit of appropriate topics [...]' (Murray and Wynne, 2001: 167). Interpreters certainly need to be versed in qualitative research methods (as was the case in our research setting) in order to help generate nuance-rich accounts about people's social realities. In our dataset, exchanges in which the interpreter asked probes to acquire more detail and clarification were particularly common. At times, the interpreter - taken aback by the brevity of Ram's responses - decided to engage in subtle elicitation. His verbal probing in Arabic precluded misinterpretation on multiple occasions and allowed for detours into new, relevant directions. During the stimulated recall interview (conducted after the first analytical inspection), the interpreter was presented with cues from the audio and transcript to evoke a vivid discussion about probing questions. Naturally, the interpreter's questions and follow-up probes were dependent on his own interpretational framework, i.e. his understanding of the (kind of) information each question in English was intended to elicit. The recall interview 
provided groundwork for exploring how (and to what extent) the interpreter's real-time reflections shaped the construction of the research account. For instance, the extract below hints at the interpreter's hesitance to recount certain sensitive and emotionally laden topics:

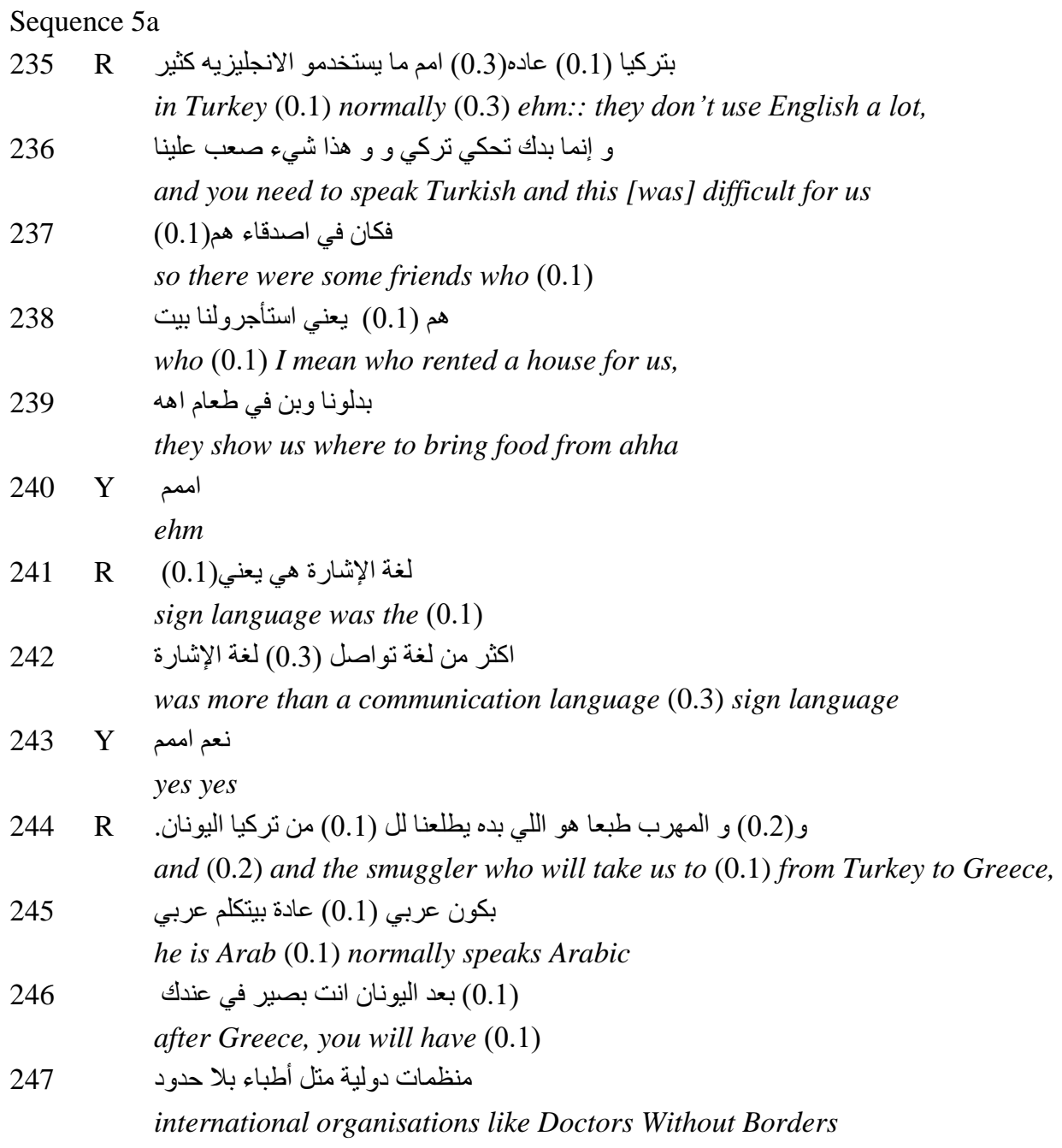

Interpreter's rendition (Sequence 5b):

$271 \mathrm{Y}$ in Turkey it was difficult for them (0.1) because they- in Turkey

272 they don't speak a lot English a:nd the refugees don't speak Turkish

273 so they had some friends who helped them

274 and they >used body language most of the time in Turkey< $(0.3)$

275 then when the:y ehm:: moved from Turkey then there were organizations

276 international organizations like ehm: (0.2) Doctors Without Borders?

In his rendition, the interpreter transmitted a sanitised version of Ram's migration path, removing the details about the Arabic-speaking smuggler who arranged his transport from the shores of Turkey to Greece. The pause and hesitations in the output (then when the:y ehm:: moved from Turkey) are indicative of a well-considered choice to withhold these details. Different explanations exist in the literature regarding this (contentious) approach, which is generally referred to as selective interpreting. For instance, an interpreter drawn from the 
same ethnic community as the interviewee(s) may be reluctant to recount experiences, ideas and practices which reflect their community in an unfavourable light (Murray and Wynne, 2001). Similarly, s/he may feel uneasy about the level of disclosure, as was (almost) certainly the case here. Although rapport-building to facilitate participant disclosure is part of the role of interpreters, 'the openness and intimacy of the interview may be seductive and lead subjects to disclose information that they may later regret' (Kvale, 1996: 116). Following this line of thought, we argue that the interpreter's decision to omit information can be regarded as a measure to protect a vulnerable research participant from (presumed) harm. Such rich points invite us to reflect on the standards that underlie our everyday choices and judgments about communication across languages.

The excerpt under consideration also brings out the prominence of repetition in dialogue interpreting. In lines 271-274 (sequence 5b), the interpreter reiterates the adverbial phrase (in Turkey) multiple times, revealing a preference for lexical over referential coherence. Similarly, in his renditions, the source text pronouns are often substituted with a fuller definite description: for example, in the extract above, the participant's comment - and و إنما بدك تحكي تركي و و هذا شيء. - was encoded in the target language as a:nd the refugees don't speak Turkish. However, we also know from the literature that the meaning of personal pronouns is context dependent and retrievable only by inference (Bull and Fetzer, 2006). Indeed, the meaning of 'you' and 'us' is so elastic that it can index an entire social group ( $\approx$ refugees). But 'us' could also have meant a different subgroup $(\approx$ immediate family). Ram fled Syria together with his wife and two children (information which was not available to the interpreter at this point of the interview); therefore, it is plausible that the exclusive we/us did, in fact, refer to them. A third distinct interpretation was proposed by the second author: in some dialects of Arabic, speakers use a plural pronoun in place of the singular pronoun 'I' أنا) to give less importance to themselves and/or to make their speech less personal. In this case, the first person plural pronouns would include (solely) the speaker $(\approx$ Ram). Examples of singular uses of 'we' were found throughout the interview:

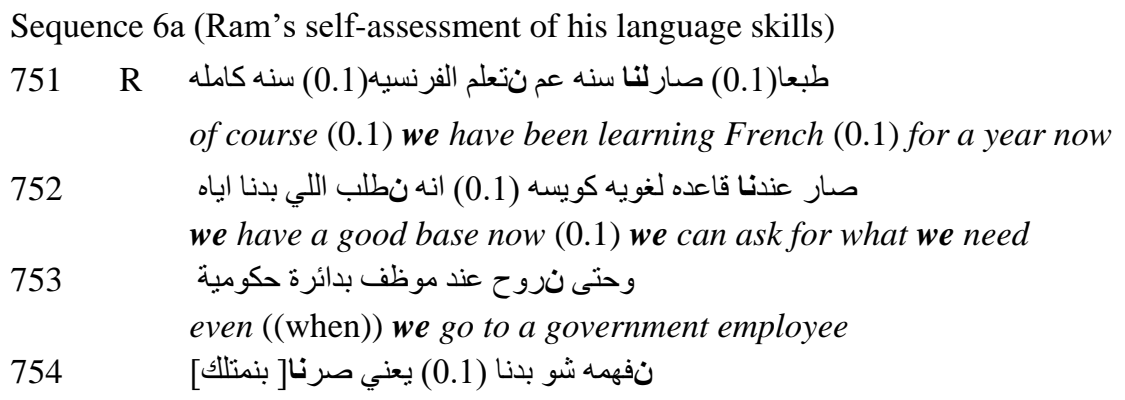



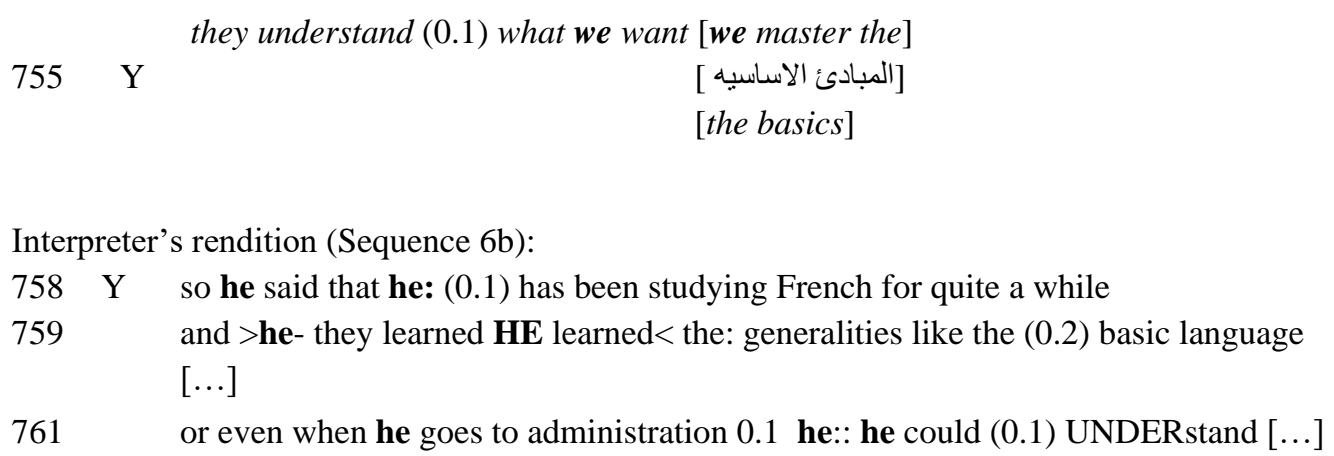

After a brief moment of hesitation (in line 759), the interpreter switched from a plural to a singular pronoun. The same - singular - sense was confirmed by the second author (note that the we-form was transferred into English for illustrative purposes).

Finally, the translation of even single words can lead to vivid debates in research teams, especially if the language of research exchange is ELF. This is well illustrated in the following example. In lines 241-242 (sequence 5a), Ram searches for an appropriate word to describe the strategies he used for overcoming language barriers. After some pauses and cutoffs, he utters the word لغة الإشارة (sign language). In his turn, the interpreter translates the same lexical item as body language, producing a subtle shift in meaning الجسد >= لغة الإشارة) لغة). Both translation solutions use terms with multiple meanings, which can introduce interpretative bias: e.g. in the researcher's mental lexicon, sign language was associated more closely with recognised manual signs and gestures (used by and to people who cannot hear or talk). This interpretation would suggest that Ram is a signer. Essentially, the same considerations led the interpreter to substitute body for sign language in his rendition. However, body language also encompasses unconscious movements and postures, thus its scope goes beyond the intended meaning, which we believe was 'communication via (intentional) gestures'. Taken together, the above examples provide nuanced insights into our processes of negotiation, in which an agreed upon sense of the source meaning was established. Our analysis also directed attention to the fluidity of interpreter-mediated research accounts and to the fact that meaning is jointly constructed, partly in the course of the research interview itself but also at the point of data representation and interpretation. A more explicit elaboration of our main observations is provided in the next section.

\section{Conclusions}

By examining the interactional dynamics of an interpreter-mediated research encounter, we set out to bring cross-language communication back into the focus of methodological 
discussions. Our contribution has built on multilingual interview material extracted from a larger ethnographic study on forced migration and multilingualism. It has considered three different but complementary analytical perspectives - common language, equivalence, and loss and gain in translation - and offered a number of detailed proposals on how to handle (or even turn into gain) the complexities of cross-language interview research. Our examples show that there is merit in fixing our analytical gaze on the minute details of language use across different codes, as these offer an entry point to broader and less immediate processes of meaning construction. Similarly, a closer examination of interactional dynamics facilitates locating and observing (otherwise elusive) moments of rapport-building, role performances and ethical unease, all of which are central to qualitative research from start to finish. Despite its limited scope, our paper adds to the rapidly expanding field of interpreting and (collaborative) translation: for example, we have exposed, interpreted and theorised some contested shifts in register, omissions, inferencing and strategies for creating inter-turn cohesion.

Our examples also provide a good starting point for investigating the amount of stake displayed by interpreters in data collection processes. An ethnometodological framework (e.g. Baker, 2002) could be well placed to further examine the ideological and methodological implications of this role shift. Finally, the favourable outcomes of our collaborative endeavour support the long-held academic view that a greater involvement on the part of the interpreter, translator and transcriber in data interpretation processes generates richer data and more accurate reporting. Since an important part of mainstream research is currently being conducted, by necessity, in more than one language, our insights can assist researchers across various fields. However, considerably more work will need to be done to establish a robust multilingual research praxis.

\section{References}

Albirini A (2014) Toward understanding the variability in the language proficiencies of Arabic heritage speakers. International Journal of Bilingualism 18(6): 730-765.

Andrews J (2013) “It's a very difficult question isn't it?” Researcher, interpreter and research participant negotiating meanings in an education research interview. Applied Linguistics 23(3): 316-328.

Baker CD (2002) Ethnomethodological analysis of interviews. In: Gubrium J and Holstein J (eds) Handbook of Interviewing: Context and Method. Thousand Oaks: SAGE Publications, pp. 777-795. 
Bassnett S (2013, $4^{\text {th }}$ ed.) Translation Studies. London: Routledge.

Bellos D (2011) Is That a Fish in Your Ear? Translation and the Meaning of Everything. New York: Faber and Faber.

Berman RC and Tyyskä V (2011) A critical reflection on the use of translators/interpreters in a qualitative cross-language research project. International Journal of Qualitative Methods 10(2): 178-190.

Brämberg BE and Dahlberg K (2013) Interpreters in cross-cultural interviews: a three-way coconstruction of data. Qualitative Health Research 23(2): 241-247.

Bull P and Fetzer A (2006) Who are we and who are you? The strategic use of forms of address in political interviews. Text \& Talk - An Interdisciplinary Journal of Language, Discourse Communication Studies 26(1): 3-37.

Dempsey NP (2010) Stimulated recall interviews in ethnography. Qualitative Sociology 33(3): 349-367.

Crystal D (2008, $6^{\text {th }}$ ed.) A Dictionary of Linguistics and Phonetics. Oxford: Blackwell Publishing.

Edwards R (2013) Power and trust: an academic researcher's perspective on working with interpreters as gatekeepers. International Journal of Social Research Methodology 16(6): 505-514.

Fersch B (2013) Meaning: lost, found or 'made' in translation? A hermeneutical approach to cross-language interview research. Qualitative Studies 4(2): 86-99.

Firth A (1996) The discursive accomplishment of normality: on 'lingua franca' English and conversation analysis. Journal of Pragmatics 26: 237-259.

Freed AO (1988) Interviewing through an interpreter. Social Work 33: 315-319.

Gibb R and Good A (2014) Interpretation, translation and intercultural communication in refugee status determination procedures in the UK and France. Language and Intercultural Communication 14(3): 385-399.

House J (2014) English as a global lingua franca: A threat to multilingual communication and translation? Language Teaching 47(3): 363-376.

Jacobsen B (2004) Pragmatic meaning in court interpreting: an empirical study of additions in consecutively interpreted question-answer dialogues. Hermes (Journal of Lingusitics) 32: $237-249$.

Jawad H (2009) Repetition in literary Arabic: foregrounding, backgrounding, and translation strategies. Meta 54(4): 753-769. 
Kalocsányiová, E. (2017) Towards a repertoire-building approach: multilingualism in language classes for refugees in Luxembourg. Language and Intercultural Communication 17(4): 474-493.

Kalocsányiová, E. (2017) At the borders of languages: the role of ideologies in the integration of forced migrants in multilingual Luxembourg. Journal of Ethnic and Migration Studies 46(9): 1903-1920.

Keselman O, Cederborg A-Ch, Lamb ME and Dahlström Ö (2010) Asylum-seeking minors in interpreter-mediated interviews: What do they say and what happens to their responses? Child \& Family Social Work 15(3): 325-334.

Kogelmann F (2010) Maghreb. In: Ende W and Steinbach U (eds) Islam in the World Today: A Handbook of Politics, Religion, Culture, and Society. Ithaca: Cornell University Press, pp. $402-414$.

Kramsch C (2014) The challenge of globalization for the teaching of foreign languages and cultures. Electronic Journal of Foreign Language Teaching 11(2): 249-254.

Kvale S (1996) InterViews: An Introduction to Qualitative Research Interviewing. Thousand Oaks: SAGE Publications.

Määttä SK (2015) Interpreting the discourse of reporting: the case of screening interviews with asylum seekers and police interviews in Finland. Translation and Interpreting 7(3): 2135 .

Mason I (2006) On mutual accessibility of contextual assumptions in dialogue interpreting. Journal of Pragmatics 38: 359-373.

Merriam SB, Johnson-Bailey J, Lee M-Y, Kee Y, Ntseane G. and Muhamad M (2001) Power and positionality: negotiating insider/outsider status within and across cultures. International Journal of Lifelong Education 20: 405-416.

Milligan L (2016) Insider-outsider-inbetweener? Researcher positioning, participative methods and cross-cultural educational research. Compare: A Journal of Comparative and International Education 46(2): 235-250.

Morris R (2010) Images of the court interpreter: professional identity, role definition and selfimage. Translation and Interpreting Studies 5(1): 20-40.

Munday J (2001) Introducing Translation Studies: Theories and Applications. London: Routledge.

Murray CD and Wynne J (2001) Researching community, work and family with an interpreter. Community, Work \& Family 4(2): 157-171.

Nida EA (1964) Toward a Science of Translating. Leiden: Brill. 
Oliver D, Serovich J and Mason T (2005) Constraints and opportunities with interview transcription: towards reflection in qualitative research. Social Forces 84(2): 1273-1289. Prior MT (2017) Accomplishing 'rapport' in qualitative research interviews: emphatic moments in interaction. Applied Linguistics Review 9(4): 1-25.

Pöchhacker F (2004) Introducing Interpreting Studies. London: Routledge.

Resch K and Enzenhofer E (2018) Collecting data in other languages - strategies for crosslanguage research in multilingual societies. The SAGE Handbook of Qualitative Data Collection. London: SAGE Publications, pp. 131-147.

Ross J (2010) Was that infinity or affinity? Applying insights from translation studies to qualitative research transcription. Forum: Qualitative Social Research 11(2). DOI: 10.17169/fqs-11.2.1357

Shklarov S (2007) Double vision uncertainty: the bilingual researcher and the ethics of cross language research. Qualitative Health Research 17: 529-538.

Straniero Sergio F (2012) Repetition in dialogue interpreting. In: Kellett CJ (ed) Interpreting across Genres: Multiple Research Perspectives. Trieste: Edizioni Università di Trieste, pp. 27-53.

Temple B and Young A (2004) Qualitative research and translation dilemmas. Qualitative Research 4(2): 161-178.

Temple B and Edwards R (2002) Interpreters/translators and cross-language research: reflexivity and border crossings. International Journal of Qualitative Methods 1(2): 1-12. Translators Without Borders (TWB) (2017) Putting Language on the Map in the European Refugee Response. Available at: https://translatorswithoutborders.org/wpcontent/uploads/2017/04/Putting-language-on-the-map.pdf (accessed 10 December 2018). Tribe R and Keefe A (2009) Issues in using interpreters in therapeutic work with refugees. What is not being expressed? European Journal of Psychotherapy and Counselling 11(4): 409-424.

von Glasersfeld E (1983) On the concept of interpretation. Poetics 12(2/3): 207-218.

Wadensjö C (1998) Interpreting as Interaction. London: Longman.

Widdowson H (2004) Text, Context, Pretext: Critical Issues in Discourse Analysis. Oxford: Blackwell.

Williamson DL, Choi J, Charchuk M, Rempel GW, Pitre N, Breitkreuz R and Kushner KE (2011) Interpreter-facilitated cross-language interviews: a research note. Qualitative Research 11(4): 381-394. 


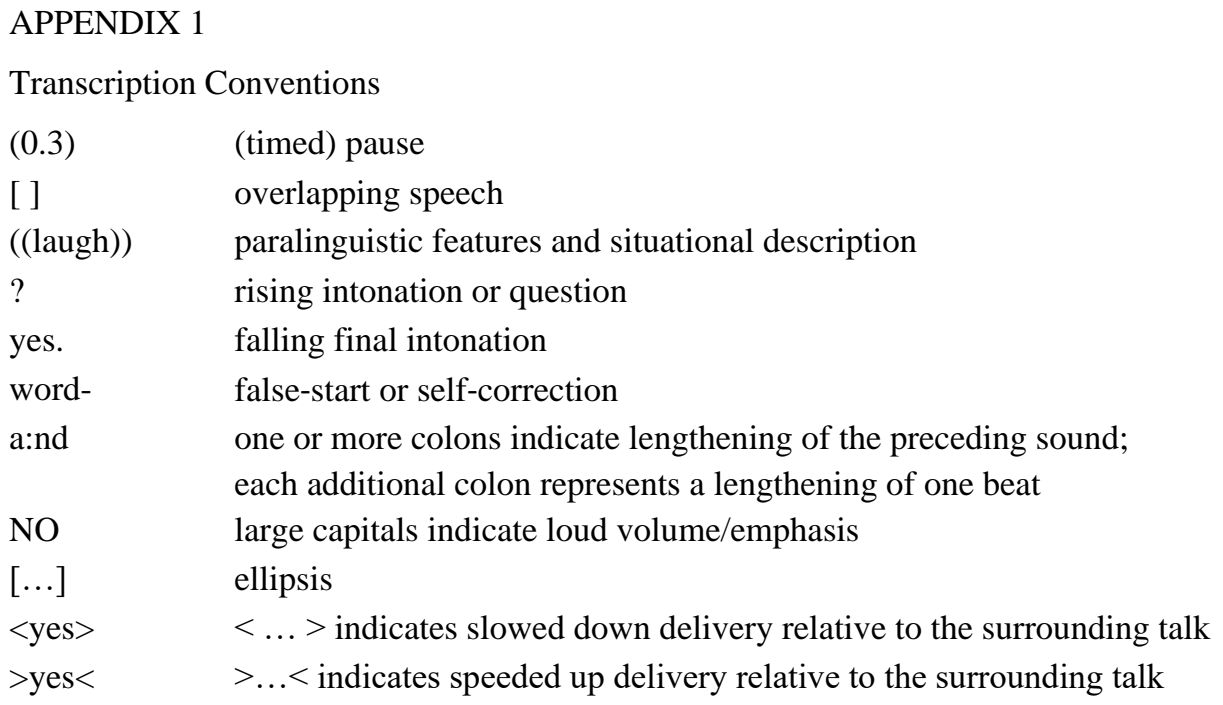

\footnotetext{
${ }^{\mathrm{i}}$ Excerpts included in this paper employ CA conventions for transcription; however, our analysis will not draw on the theory, classic topics or interpretative procedures of conversation analysis.

${ }^{\text {ii }}$ ELF as a term was used to describe the interview participants as 'language user[s] whose real-world interactions are deserving of unprejudiced description' rather than as 'possessor[s] of incomplete or deficient communicative competence [...]' (Firth, 1996: 241).

iii In sociolinguistics and stylistics, register refers to 'a variety of language defined according to its use in social situations' [emphasis in the original] (Crystal, 2008: 409).

iv The earliest theories, dating back to Horace, Cicero and St. Jerome, drew a distinction between two types of translation:

- word-for-word or literal translation, which adheres to the source text's word order and grammar, and

- sense-for-sense or free translation.
}

Interestingly, faithfulness in translation scholarship has come to mean both word-for-word transmission, and a freer, idiomatic method in passing on intended meanings from the source language to the translation.

' Formal correspondence 'focuses attention on the message itself, in both form and content'; dynamic equivalence is based upon 'the principle of equivalent effect', in which 'the relationship between receptor and message should be substantially the same as that which existed between the original receptor and the message' (Nida, 1964: 159). 


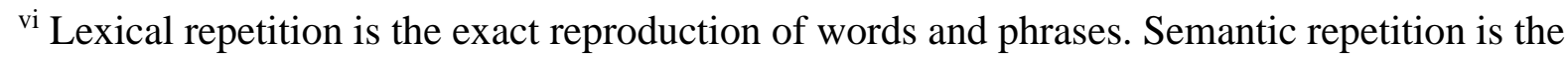
reproduction of the same meaning (theme) through synonyms. 